Artículo de investigación

Apuntes del CENES

ISSN 0120-3053

Volumen $34-\mathrm{N}^{\circ} .60$

julio-diciembre 2015

Págs. 15-40

\title{
Planteamientos sobre la pobreza: una aproximación conceptual
}

\author{
Propositions on poverty: \\ a conceptual approach
}

Abordagens sobre a pobreza:
uma abordagem conceitual

Bertha Lucía Martínez Bernal*

Fecha de recepción: 26 de enero de 2015

Concepto de evaluación: 26 de marzo de 2015

Fecha de aprobación: 25 de junio de 2015

Economista y magíster en Economía, Universidad Pedagógica y Tecnológica de Colombia, UPTC. Profesional en Asociatividad y Proyectos del Ministerio de Agricultura-UNAD. Correo electrónico: lumar770@hotmail.com 


\section{Resumen}

En el presente artículo se presentan varias definiciones o conceptos para la comprensión, medición y el análisis de la pobreza. Para ello se retoma la producción escrita de diferentes estudiosos e investigadores que han hecho aportes valiosos sobre el tema. Al lado de estos conceptos se analizan los diferentes enfoques que se utilizan para aproximarse al estudio de la pobreza, aunque estas no son teorías ni explicaciones de sus causas, sino perspectivas sobre pobreza que denotan dichos enfoques. Así mismo se desarrolla una revisión de las metodologías más utilizadas en los procesos de identificación y agregación. Finalmente se interpretan las políticas públicas en los sectores sociales.

Palabras clave: pobreza, equidad social, política social, desigualdad, métodos de medición, necesidades básicas.

Clasificación JEL: D60, I31, I32, I38.

\section{Abstract}

In this article are presented several definitions or concepts for understanding, measuring and analyzing poverty. For that written production of different scholars and researchers who have made valuable contributions on the subject is taken. Beside these concepts are analyzed different propositions used to approach the study of poverty, although these are not theories or explanations of its causes, but perspectives on poverty denoting such approaches. Likewise it develops a review of the methodologies used in the processes of identification and aggregation. Finally public policies in the social sectors are interpreted.

Keywords: poverty, social equity, social policy, inequality, measurement methods, basic needs. 


\section{Resumo}

Neste artigo várias definições ou conceitos para a compreensão, medição e análise da pobreza, pois é a produção literária de diferentes estudiosos e pesquisadores que fizeram contribuições valiosas sobre o tema, ao lado desses conceitos são apresentados currículos onde são analisadas as diferentes abordagens utilizadas para abordar o estudo da pobreza, não sendo estas teorias ou explicações sobre as suas causas, mas as perspectivas sobre a pobreza que denota essas abordagens, igualmente uma revisão das metodologias utilizadas nos processos de identificação e agregação. Finalmente, a ação da política pública é interpretada nos sectores sociais.

Palavras-chave: pobreza, igualdade social, política social, desigualdade, desenvolvimento, necessidades básicas. 


\section{INTRODUCCIÓN}

Este artículo busca dar evidencia del estudio realizado en el trabajo de grado titulado La equidad social y la evolución de la pobreza en Boyacá y su capital para el período (1993-2005), y con el cual se estudió la economía de la pobreza, concretamente, para detectar el número de pobladores de nuestro departamento afectados por este flagelo y las políticas del Gobierno para disminuir la pobreza para el caso específico de Boyacá y su capital.

Los altos niveles de pobreza y de desigualdad en una región pueden estar asociados con bajos niveles de actividad económica, mientras que regiones con mayor participación económica presentan tasas de pobreza menores. Aunque el comportamiento de la línea de indigen- cia (LI) presente dinámicas diferentes, que reflejan reducciones graduales, por la existencia de empleos precarios, los cuales permiten a la población superar la línea de indigencia, pero no lograr salir de la línea de pobreza; hecho que convierte este tema en una prioridad para los gobiernos nacionales, departamentales y municipales.

En efecto, el primer Objetivo de Desarrollo del Milenio insta a los países comprometidos a reducir a la mitad el nivel de pobreza hacia 2015. Muchas políticas públicas se han orientado a reducir el porcentaje de personas en condiciones de vida precarias, con especificaciones particulares en cada país, departamento o municipio. Es así que este es un tema de gran relevancia y que continúa dando lugar a avances tanto en términos de desarrollo académico como práctico. Hacia finales 
del siglo anterior se enfatizó en la literatura sobre pobreza, y los diferentes estudiosos aportaron conceptos y formas de medirla. De hecho, un individuo puede enfrentar privaciones en diversas dimensiones de su vida, como en su educación, capacidad de obtener un empleo y condiciones de salud, vivienda, que deben ser contempladas en las mediciones de pobreza. Si bien existen mediciones que van más allá del ingreso, como las basadas en NBI, en general, las estimaciones oficiales y las metas de política se fijan en términos de reducir el porcentaje de individuos (u hogares) con ingresos por debajo de un determinado umbral. Pese a esto, existe consenso de que la pobreza es multidimensional y que las futuras mediciones deberían orientarse a ampliar la noción de pobreza.

Efectivamente, el tema de la pobreza es un tema de una amplia significación, que da origen a avances en términos de desarrollo académico y práctico. Con el avance del tiempo se ha venido enfatizando en la literatura sobre la pobreza en el aspecto multidimensional. Es ampliamente aceptado que las tradicionales estimaciones de pobreza basadas únicamente en privaciones de ingreso o necesidades básicas insatisfechas (NBI) resultan limitadas, dado que la pobreza va más allá de estas definiciones. Realmente, un individuo puede enfrentar privaciones en diversas dimensiones de su vida, como en su educación, capacidad de obtener un empleo y condiciones de salud, que no son contempladas en ninguna de las mediciones tradicionales de pobreza.
Por lo anterior, el estudio realizado en este artículo se enmarca dentro de los conceptos de pobreza, establecida por diferentes autores y entidades, y las formas de medirla, junto con las distintas políticas sociales del Gobierno en busca de eliminar o por lo menos disminuir los niveles de pobreza. En este sentido, el presente documento se concentrará en planteamientos de diferentes autores que reflejan los niveles de pobreza.

\section{PLANTEAMIENTOS DIRIGIDOS A LA DISMINUCIÓN DE LAPOBREZA}

Haciendo una revisión histórica se nota que durante los últimos 50 años, a medida que se ha llegado a una mayor comprensión de la complejidad del proceso de desarrollo, los estudiosos de este consideraban que la realización de inversiones de gran importancia en capital físico e infraestructura era el medio más eficaz para impulsar dicho proceso de desarrollo.

Es así como a partir de 1970 se comenzó a dar una conciencia más clara de que no era suficiente crear solamente capital físico, y de que las mejoras en la salud, educación y vivienda merecían gran importancia. Estas nuevas ideas se plasmaron en el Informe sobre el Desarrollo Mundial de 1980, en el que se alegaba que era importante mejorar los niveles de salud y de educación, no solo por su significado intrínseco, sino también en cuanto instrumento para aumentar los ingresos de la población pobre. 
Durante los años ochenta se produjo un nuevo cambio de rumbo a causa de la crisis de la deuda y la recesión mundial y de las diferentes experiencias de los países de Asia oriental, por una parte, y de América Latina, Asia meridional y África al sur del Sahara, por la otra. El resultado fue que se hizo más hincapié en mejorar la gestión económica y dar más rienda suelta a las fuerzas del mercado. En el Informe sobre el Desarrollo Mundial de 1990, en relación con la pobreza, «se propuso una doble estrategia: por un lado, promoción de un crecimiento basado en el uso intensivo de mano de obra mediante la apertura de las economías y la inversión en infraestructura, por el otro, mejoramiento del acceso de los servicios sociales básicos de salud y educación a la población más pobre» (Informe sobre el Desarrollo Mundial, 2000, p. 77). En dicho informe se propone una estrategia de lucha contra la pobreza basada en la adopción de medición en tres esferas: oportunidad, potenciamiento y seguridad.

En septiembre de 1990 en Quito (Ecuador), se llevó acabo la Conferencia Regional de los Países de América Latina sobre la Pobreza. El Proyecto Regional ONU de Superación de la Pobreza estimó que había en situación de pobreza 270 millones de latinoamericanos, es decir, cerca del $62 \%$ de la población. Este informe indica que siendo el crecimiento económico central para enfrentar el problema social, de ningún modo lleva al progreso social; puede haber crecimiento, pero las desigualdades y políticas sociales débiles llevan a que no se difunda y el problema social siga agravándose. E1 informe cuestiona también los métodos muy esquemáticos con que se mide el desarrollo cuyo objetivo final es que la gente viva más años y mejore su calidad de vida. Por lo tanto se debe superar las mediciones con base en el ingreso per cápita e incluir indicadores como esperanza de vida, escolaridad, participación social, entre otros. Habrá desarrollo real cuando ese conjunto de indicadores mejoren efectivamente.

En la Conferencia Mundial sobre Management Social de Copenhague (julio de 1991) existió total consenso sobre este tema de derechos humanos básicos. No se trata de hacer caridad, sino de establecer que la sociedad es responsable de garantizar a sus miembros posibilidades de trabajo y satisfacción de sus necesidades esenciales. De hecho, en la Cumbre Mundial de Desarrollo Social de Copenhague, la ONU (1995, p. 45) definió la pobreza absoluta como «condición caracterizada por una privación severa de las necesidades humanas básicas, que incluyen alimentos y agua potable, así como condiciones de higiene, salud, vivienda, educación e información».

En igual dirección se pronunció la última reunión del Club de Roma (1991), reconociendo la pobreza como el gran problema que tiene por delante la humanidad, denunciando las diferencias económicas, la extrema pobreza enfrentada al exceso de riqueza, la insolidaridad de los países ricos para con los pobres y la necesidad de combatir las diferentes 
muestras de egoísmo que se manifiestan tanto en el terreno material como en el espiritual.

En la década de los noventa se reconoció que los programas sociales compensatorios constituyen un instrumento paliativo ineludible en situaciones extremas, pero que no representan un aporte significativo en el combate contra la pobreza, ya que este requiere programas que provean «herramientas» a los sectores pobres para que salgan de la pobreza con su propio esfuerzo y programas gubernamentales que contribuyan a la creación de oportunidades efectivas de integración social.

Después de estas conferencias, los gobiernos nacionales han implementado programas y políticas dirigidos al mejoramiento del desarrollo social. Así mismo, estas conferencias han dado como resultado que las Naciones Unidas den una nueva orientación a sus actividades.

\section{CONCEPTUALIZACIÓN DE LA POBREZA}

En el presente apartado se describen diferentes conceptos de pobreza y de pobreza extrema. Existen variadas definiciones de pobreza, ya que la amplia literatura que habla sobre esta, deja ver que no existe un concepto unívoco sino que, por el contrario, existen variadas nociones: se inicia definiendo la pobreza como una situación compleja y multidimensional ${ }^{1}$. Debido a lo anterior existen múltiples definiciones y maneras de medirla, y cada país tiene un estándar para calcularla. Una definición muy común de pobreza es la privación material, medida mediante el ingreso o el consumo del individuo o la familia.

Además de los conceptos anteriores, la CEPAL indica que bajo el término de «pobreza» son abarcados diversos aspectos de la vida social y humana, valorados negativamente; se destacan dos características dentro del concepto de pobreza:

Es un concepto multidimensional: esto abarca un síndrome situacional al cual están asociadas circunstancias como: desnutrición, bajos niveles educativos, inserción inestable en la producción o dentro de sectores atrasados de la misma, condiciones habitacionales y sanitarias precarias. En una perspectiva amplia no solo se refiere a la insatisfacción de necesidades básicas materiales, sino que se extiende a otras necesidades también básicas aunque de naturaleza no material: autorrealización personal, libertad, derechos humanos, participación en mecanismos sociales de integración y decisión y en las manifestaciones culturales (CEPAL, 2001, p. 12).

\footnotetext{
Cuando se mencionan aspectos multidimensionales se hace referencia a muchos factores: educación: 1) bajo logro educativo, 2) analfabetismo. Niñez y juventud: 3) inasistencia escolar, 4) rezago escolar, 5) no acceso a servicios relacionados con larga duración, 8) tasa de ocupación. Salud: 9) no aseguramiento, 10) no acceso a servicios de saludad dada una necesidad: 11) no acceso a fuentes de agua mejorada, 12) eliminación de excretas, 13) pisos inadecuados, 14) paredes inadecuadas, 15) hacinamiento crítico.
} 
«Una persona es considerada pobre si él o ella no tiene acceso (o no posee la capacidad para acceder) a un paquete de bienes, servicios y derechos establecidos normativamente» (CEPAL, 1997).

Es un concepto relativo a un contexto histórico y social determinado: se basa en juicios de valor sobre los niveles de bienestar mínimamente aceptables para llevar una vida digna, sobre las necesidades básicas, cuya satisfacción es indispensable, y sobre los grados de privación que se consideran intolerables. La apreciación acerca de la pobreza, evoluciona a medida que progresa la sociedad y varía con las condiciones materiales y culturales.

El concepto de pobreza se ha desviado a un marco teórico como un concepto analítico relevante; se ajusta más a una categoría descriptiva que revela un fenómeno observable y medible. Su utilización es enriquecedora en la descripción de las desigualdades sociales y cobra especial importancia cuando existe la voluntad política de erradicar las situaciones críticas de insatisfacción de las necesidades humanas básicas.

Una de las definiciones del Banco Mundial sobre la pobreza es «la incapacidad para alcanzar un nivel de vida mínimo» (Banco Mundial, 1990, p. 27). Este organismo estudia la contribución al nivel de vida de bienes y de servicios públicos así como los recursos de propiedad de una comunidad. Instaura un nivel «basa- do en el consumo», el cual incluye dos elementos: el primero, «el gasto necesario para acceder a un estándar mínimo de nutrición y otras necesidades muy básicas»; y el segundo, "una cantidad que varía de un país a otro y que refleja el costo que tiene la participación en la vida diaria de las sociedades» (Banco Mundial, 1990, p. 26).

Es así que el Banco asevera que el gasto necesario es «relativamente sencillo», porque puede ser calculado «observando los precios de los alimentos que forman las dietas de los pobres». Sin embargo, el segundo elemento es «mucho más individual; en algunos países, el acceso a las cañerías en las viviendas del hogar es un lujo, pero en otros es una 'necesidad'»(Banco Mundial, 1990, p. 27). Con fines operacionales, el segundo elemento (mencionado anteriormente) se dejó de lado y el primero se valoró como paridad de poder adquisitivo (PPA) (370 dólares anuales por persona, para todos los países en desarrollo más pobres). Se clasifican como «pobres» aquellos individuos que tienen un ingreso per cápita menor a 370 dólares, (población que vive con menos de US\$1.08 diario, precios internacionales de 1993) mientras que aquellos con menos de 275 dólares al año eran «extremadamente pobres».

El esquema es sencillo y fácil de aplicar. No depende de la recolección y compilación inflexible y continua de datos acerca de los tipos y cantidades de recursos, de los cambiantes estándares de necesidades y de las estimaciones variables de 
los niveles de vida. Además, no es verdaderamente «global», puesto que solo es adaptable para los países más pobres. Si bien el Banco Mundial ha reconocido la necesidad de una línea internacional de pobreza que esté «basada más allá del consumo», no la ha desarrollado ni se ha estimado el costo para el segundo elemento «participativo» de la definición.

En el mismo informe se encuentra también una definición de pobreza basada en el cálculo del producto bruto nacional per cápita $(\mathrm{PBN})$, completado por otros criterios como consumo per cápita, mortalidad infantil en menores de cinco años, esperanza de vida al nacer y matrícula escolar primaria.

El Banco Mundial ha iniciado una serie de evaluaciones de la pobreza en países específicos y conceptualiza la pobreza en forma cuantitativa al afirmar que una persona es pobre cuando tiene menos de dos dólares al día, datos que se recogen en forma cuantitativa y cualitativa, a través de encuestas de hogares, perfiles de pobreza, estudios participativos, evaluaciones de beneficiarios, análisis del gasto público, análisis económicos nacionales y análisis sectoriales.

Las valoraciones sobre la pobreza son importantes para identificar las dificultades para cada país y dar cuenta de las diferentes definiciones de pobreza.

Los anteriores conceptos sobre la pobreza han venido cambiando, la pobreza como fenómeno multidimensional alude al fenómeno de la pobreza desde un enfoque mucho más completo, ya que incorpora otros factores, además del meramente monetario (ingreso). Surge el estado de la vivienda, salud, educación, empleo, carencias relacionadas con el desarrollo humano, tales como libertad, dignidad, autoestima, es decir, los derechos de primera, segunda y tercera generación, donde todos estos elementos están directamente correlacionados entre sí.

El Oxford Poverty \& Human Development Initiative (OPHI) ha desarrollado el índice de pobreza multidimensional (IPM), como el indicador que refleja el grado de privación de las personas en un conjunto de dimensiones; la medida permite determinar la naturaleza de la privación -de acuerdo con las dimensiones seleccionadas- $\mathrm{y}$ la intensidad de la misma. El IPM es la combinación del porcentaje de personas consideradas pobres, y de la proporción de dimensiones en las cuales los hogares son, en promedio, pobres. (Dirección de Desarrollo Social - DDS-, 2011, p. 11).

\section{POBREZA EXTREMA}

Para la CEPAL, la «pobreza extrema» o «indigencia» se entiende como la situación en que no se dispone de los recursos que permitan satisfacer al menos las necesidades básicas de alimentación. En otras palabras, se considera como «pobres extremos» a las personas que residen en hogares cuyos ingresos no alcanzan para adquirir una canasta básica de alimentos, 
así lo destinaran en su totalidad a dicho fin. A su vez, se entiende como «pobreza total» la situación en que los ingresos son inferiores al valor de una canasta básica de bienes y servicios, tanto alimentarios como no alimentarios.

Para autores como Boltvinik (1990), quien hace referencia a este término como la situación de aquellos hogares que aunque destinan todo su ingreso a la alimentación, no pueden satisfacer sus necesidades básicas.

Se habla de pobreza extrema o pobreza absoluta como la falta de ingreso necesario para satisfacer las necesidades de alimentación básicas; sin embargo, uno de los conceptos de mayor aceptación es el que plantea Amartya Sen, quien manifiesta que:

el primer requisito para conceptuar la pobreza es tener un criterio que permita definir quién debe estar en el centro de nuestro interés. Especificar algunas «normas de consumo» o una «línea de pobreza» puede abrir parte de la tarea: los pobres son aquellos cuyos niveles de consumo caen por debajo de estas normas, o cuyos ingresos están por debajo de esa línea. Pero esto lleva a otra pregunta: ¿el concepto de pobreza debe relacionarse con los intereses de: 1) sólo los pobres; 2) sólo los que no son pobres, o 3) tanto unos como otros? (Sen, 2009, p. 21).

Así mismo se analizan los diferentes enfoques de superación de la pobreza; uno de ellos es el basado en los ingresos: el cual hace referencia al estudio de la pobreza vista desde la carencia de ingresos. Elevadas tasas de desempleo inciden sobre los niveles de pobreza, ya que impiden a la familia devengar ingresos para el acceso a bienes y servicios indispensables y así llevar una vida digna. Estar desempleado puede generar una desacumulación de activos y el consumo de las medidas de aseguramiento de los hogares contra posibles contingencias, tales como el ahorro, la vivienda y otros activos, y puede causar la desafiliación a la seguridad social en salud.

Otras variables que guardan una relación directa con los niveles de pobreza son el crecimiento de los precios (frente al de los ingresos), que repercute sobre la posibilidad de adquirir la canasta de bienes y servicios, y tipo de cambio que afecta los ingresos y los costos de los bienes exportados e importados, así como el precio de los bienes transables, entre ellos, los alimentos.

\section{LAS DIMENSIONES DE LA PO- BREZA}

Dentro de las dimensiones tenidas en cuenta para el estudio de la pobreza se pueden destacar dos fundamentales: los estudios realizados con una variable unidimensional, referida al bienestar alcanzado por los individuos, y variables de carácter multidimensional, en las que se utiliza un indicador de recursos como la renta, la riqueza o el gasto; y los estudios que se realizan sobre el 
tema de la pobreza por ser de carácter multidimensional, como los llevados a cabo actualmente sobre la pobreza. Por lo tanto y teniendo en cuenta estudios hechos sobre la pobreza como un fenómeno multidimensional, es posible cuestionar algunas de las limitaciones de los métodos unidimensionales, cuando dichos métodos son la única alternativa práctica para llevar a cabo la realización de dichos estudios.

Teniendo en cuenta que en la literatura de la pobreza sobresale el fenómeno multidimensional (Boltvinik, 2010, p. 48), realmente no se le ha dado la suficiente importancia para realizar de forma estricta y profunda los estudios que conduzcan al logro de cada una de esas dimensiones.

Haciendo una revisión de las dimensiones que más influyen sobre los niveles de pobreza multidimensional, que son la situación laboral, referida esta al nivel educativo asociado al análisis de la calificación de la ocupación y relacionado con el tipo de contrato que tiene un individuo, un estado de salud adecuado, y demás variables, que puedan ser tenidas en cuenta.

\section{ENFOQUE DE POBREZA BASADA EN CAPACIDADES}

Amartya Sen (1996) plantea el enfoque sobre capacidades (CA), el cual está ampliamente aceptado en el ámbito del desarrollo. Hay cuestionamientos relativos a la aplicación práctica de dicho enfo- que dentro de los límites en los que se mueven los participantes, profesionales y otros interesados en el estudio, en el momento de diseñar e implementar programas de reducción de la pobreza. Sen (1996) se refiere a las «capacidades» como todo aquello que le permite a una persona estar bien nutrida, escribir, leer y comunicarse, tomar parte de la vida comunitaria. Sen señala que también forman parte de estas «capacidades» las necesidades básicas, que se refieren a algo mucho más amplio. El número de opciones que las personas tienen y la libertad de elección sobre estas opciones también contribuye al bienestar humano. De esta forma, desde el enfoque de las capacidades, se afirma que más libertad y más capacidad de elección tienen un efecto directo sobre el bienestar, incrementándolo constantemente.

En primer término, se dice que existe bienestar humano cuando las personas logran desarrollar sus capacidades. Por lo tanto, se puede hablar de desarrollo cuando las personas son capaces de hacer más cosas por sí mismas, no cuando estas son capaces de adquirir más bienes o servicios en compra. El desarrollo humano se ha definido como un enfoque amplio cuyo objetivo es ampliar la gama de libertades y opciones de las personas. Al dar prioridad a las técnicas de valoración participativas basadas en el enfoque de capacidades durante el diseño y la implementación de programas de reducción de la pobreza, se abre la posibilidad de lograr efectos de gran alcance sobre las ideas de desarrollo en las que el foco 
se sitúa continuamente en la mejora de las capacidades, las libertades y el concepto de capacidad individual.

En segundo lugar, Sen estableció que existen cuestiones de género, las cuales son parte integral de los procesos de desarrollo. Descalificó que bajos niveles de desarrollo económico afectasen de igual manera a hombres y a mujeres y que las políticas de desarrollo sean neutrales en cuanto al género.

«El hambre no se produce por una insuficiente producción de alimentos. Las hambrunas pueden ser los resultados de una insuficiente producción, pero esta es consecuencia de unos pobres o injustos mecanismos de distribución» (Sen, 1996, p.28). Así, Sen señala que los problemas de distribución son en mayor medida la causa del hambre, contradiciendo los principios de la teoría económica tradicional.

El Programa de las Naciones Unidas para el Desarrollo (PNUD) ha venido desarrollando una serie de estudios que conllevan la conceptualización de la pobreza centrada en la vida real de las personas, y elaboró un procedimiento para medir internacionalmente la «pobreza de capacidades» (PNUD, 1997). Dado que este intento de realización no ha dado una respuesta satisfactoria, debido a que no ofrece un listado consistente de capacidades humanas básicas, así como tampoco de umbrales mínimos de realización en cada espacio de evaluación. El problema de la pobreza se define parale- lamente a la denegación de opciones y oportunidades fundamentales del desarrollo humano: como lo es vivir una vida larga, sana y creativa y lograr disfrutar de un nivel decente de vida, libertad, dignidad, respeto por sí mismo y por los demás (PNUD, 1997). Con todo esto los estudios desarrollados por el Programa de las Naciones Unidas para el Desarrollo (PNUD) acaban por resolver el problema de manera más tradicional, combinando métodos de ingresos o de necesidades básicas, aceptando de hecho la relatividad en los parámetros de déficit en materia de desarrollo humano.

\section{CAPITAL HUMANO Y CAPACI- DAD HUMANA}

El proceso investigativo acerca de temas sociales se ha venido desarrollando durante los últimos años, asentándose en una posición normativa acerca de la justicia global que sin desconocer los aportes brindados por las teorías de las necesidades y capacidades del desarrollo humano, promueve un enfoque de la pobreza basado en los derechos humanos. Debido a la gran importancia que tiene el capital humano, el principal objetivo de la economía es atender y ayudar a aliviar la pobreza, dándose una íntima relación entre las inversiones del Gobierno en capital humano y el alivio de la pobreza.

Durante el pasado siglo (S. XX), la educación, los talentos y otros conocimientos se han convertido en factores cruciales y determinantes 
de la productividad de una persona y de una nación. El siglo XX puede llamarse, incluso, el siglo de la edad del capital humano, en el sentido de que el factor determinante principal de la calidad de vida de un país era la medida de su éxito en el desarrollo y utilización de los talentos, conocimientos, salud y costumbres de su población (Becker, 1983, p. 82)

A partir de lo anterior, la Organización Mundial de la Salud (OMS) y la Organización Panamericana de la Salud (OPS) vienen realizando estudios que han establecido que pocos países de la región cuentan con los recursos informativos, científicos, humanos, financieros e institucionales que se necesitan para analizar y promover en forma adecuada las inquietudes de salud y ambiente en la toma de decisiones de desarrollo. Dichos estudios examinaron la disponibilidad de recursos tangibles, además de requisitos tales como las leyes, los mecanismos intersectoriales, las delegaciones funcionales de autoridad a los gobiernos municipales y la posibilidad de hacer cumplir las normas y los reglamentos. Uno de los descubrimientos de importancia crítica que se hicieron fue el de la incapacidad efectiva de casi todas las autoridades de salud nacionales para participar en el análisis y el control de los problemas de salud y ambiente a nivel sectorial o intersectorial.

Además de estos organismos, diferentes autores vienen realizando otras contribuciones en esta línea, como Pogge (2005), quien aplica el enfoque de los derechos humanos en su ensayo titulado Florecimiento humano y justicia universal, en el que adopta un criterio de justicia nuclear y sostiene la conveniencia de un criterio moralmente admisible de justicia, fundado en los «medios comunes», siendo para ello especialmente apropiado el lenguaje de los derechos, de ahí que haya una total aceptación internacional. Afirma que un principio esencial de equidad desde el enfoque de los derechos humanos, favorece la atención y la consideración de las diversas culturas del mundo y el desarrollo de una crítica característica, aunque constructiva, del orden institucional nacional e internacional, aproximándose al combate de la pobreza en el mundo (Pogge, 2005, p. 72-73).

Igualmente, Nussbaum relaciona la función de «terreno de acuerdo» que desempeña el lenguaje de los derechos humanos en el discurso público internacional, mientras se continúa discutiendo acerca del tipo de análisis apropiado en el nivel más específico (Nussbaum, 2002, p. 149).

Autores como Amartya Sen, dentro de sus estudios de la pobreza, hacen una reflexión dirigida al enfoque del desarrollo humano, en el que la acumulación del «capital humano» es su centro de análisis y lo fija en la acumulación y en la expansión de la «capacidad humana». El primer concepto se encuentra en el carácter de agentes (agency) de los seres humanos, que por medio de sus habilidades, conocimientos 
y esfuerzos, aumentan las posibilidades de producción, y el segundo se centra en su habilidad para llevar el tipo de vida que consideran valiosa e incrementar sus posibilidades reales de elección. Ambas perspectivas están relacionadas porque se ocupan del papel de los seres humanos y en particular de las habilidades efectivas que estos logran y adquieren.

\section{LAS DISTINTAS CARAS DE LA POBREZA}

En este aspecto, Amartya Sen muestra que el poder protector de la democracia para proporcionar seguridad va más allá de la prevención de las hambrunas. Tal vez la actuación de la democracia no sea siempre especialmente eficaz desde el punto de vista económico, pero sí muy importante cuando la crisis amenaza y los económicamente desposeídos necesitan protección directa de esta.

Por eso, al tener una voz de esperanza en el futuro, se aumenta cada vez más una exigencia manifiesta de democracia en el mundo y, por lo tanto, una mayor necesidad de justicia social. Se debe tener una perspectiva de la pobreza suficientemente amplia como para estar seguros de que los pobres tienen una razón para la esperanza.

Según Amartya Sen, la pobreza, es

absoluta en el plano de las capacidades y relativa en el plano de los productos básicos. En respuesta a la pregunta sobre la redefinición de la línea de pobreza para reflejar un mayor balance entre características absolutas y relativas al dar mayor peso a las medidas de las capacidades, se aclaró que la pobreza es el fracaso de un individuo para lograr un conjunto relevante de un inadecuado empleo de los recursos (Sen, 1992, p.40).

Amartya Sen ve el problema social que emana de la pobreza como un enfoque interesante, y propone una dimensión de la pobreza que los métodos y definiciones tradicionales no consideran. Es particularmente interesante que un economista premio Nobel plantee esta nueva dimensión, que, en concreto, es la ampliación de las capacidades de los individuos que les permiten hacer y desarrollarse como individuos, y no definirlos por la cantidad de dinero que poseen individual o familiarmente, lo cual les permitiría comprar determinada cantidad de bienes o acceder a ciertos servicios considerados como básicos, sino que nos permite ahondar en aspectos tales como la calidad de la educación, el nivel de desarrollo intelectual del individuo y, ¿por qué no? Ir más allá del simple concepto burgués de la «libertad y democracia», para que los pobres tengan no solo derecho a protestar y votar en cada elección de sus autoridades, sino capacidad crítica y reflexiva que les permita participar activamente en las decisiones de Estado que afectan su calidad de vida y expectativas de desarrollo.

Con lo anterior se puede aseverar que la pobreza va más allá de lo material y las 
necesidades inmediatas, pues además hay que tener en cuenta aspectos tan importantes como el desarrollo de las capacidades y de una personalidad definida del individuo que le permita tener un grado de conciencia que lo dirija a una mayor participación y desarrollo de sus habilidades, que lo ubique en el lugar donde logre su máximo progreso, lo cual se reflejará en un aumento tanto de la productividad individual como de la familiar y de la sociedad en su conjunto; pero tampoco se puede dejar de lado que para lograr esto deben existir condiciones mínimas para el avance de los individuos, como son: acceso a educación y salud de calidad y adecuada nutrición infantil.

ENFOQUE DE POBREZA DESDE EL CAPITAL SOCIAL

Bajo este enfoque es más difícil acercar el concepto de pobreza, fijando a este la importancia de las relaciones sociales. Una persona es pobre cuando no logra en la relación con su entorno mejorar sus condiciones de vida, acceder a nuevas oportunidades, ni obtener beneficios.

El capital social ocupa un lugar central en un conjunto de activos como son capital humano (activos que una persona posee por las características propias de su condición humana: conocimiento, salud, destrezas, tiempo y otros), capital social (activos que se tienen derivados de las relaciones de una persona con otras y por su participación en organizaciones), capital productivo (activos físicos: tierra, propiedades, bienes y activos financieros), capital natural (activos en forma de calidad y cantidad de recursos naturales a los que se tiene acceso), capital cultural (recursos y símbolos que se tienen como resultado de la cultura de la cual se es parte).

Desde la óptica del capital social, poner a la pobreza en el centro de la preocupación de las políticas públicas puede influir fuertemente en las posibilidades de superarla, porque permite cambiar la amplitud y naturaleza de las relaciones entre los sectores pobres y aquellos que no lo son, es decir, puede modificar la amplitud de las redes sociales y el grado de asociatividad existente entre grupos con distinto tipo de capital social.

Se destaca la importancia del capital social en la forma de vida de las personas pobres en la medida que les permite alcanzar otros recursos, fomentar su participación en organizaciones, y les ayuda a salir de su situación de pobreza, ampliando su ciudadanía y su inclusión social.

\section{ENFOQUE DE POBREZA BASADA EN LA VULNERABILIDAD SOCIAL}

La noción de vulnerabilidad tiene como potencialidad contribuir a identificar individuos, hogares y comunidades que, por su menor dotación de activos y diversificación de estrategias, están 
expuestos a mayores niveles de riesgo por alteraciones significativas en el plano social, político y económico que afectan sus condiciones de vida. Planteada de este modo, la noción de vulnerabilidad excede, a la vez que incluye, la dimensión de ingresos que tradicionalmente se ha medido desde la noción de pobreza. La noción de riesgo implícita en la noción puede observarse a través de desviaciones respecto a los valores medios o promedios de una sociedad, o bien por coeficientes de variación de algunas variables clave, como el ingreso, el empleo y el consumo de bienes y servicios. «La noción de vulnerabilidad es entendida como un proceso multidimensional que confluye en el riesgo o probabilidad del individuo, hogar o comunidad de ser herido, lesionado o dañado ante cambios o permanencia de situaciones externas y/o (sic) internas» (CEPAL, 2001, p.56)

Bajo este concepto de vulnerabilidad se intenta comprender la pobreza como el resultado de un proceso en el cual un hogar, un individuo o una comunidad se ven afectados al enfrentarse a una situación particular que deteriora su bienestar. Para entender el alcance de este enfoque, se demuestra que una familia es más vulnerable si solo uno de sus miembros trabaja, al perder esa fuente de ingreso de inmediato se afecta el nivel de vida de toda la familia. Así mismo, una persona que trabaja sin contrato laboral es vulnerable a caer en situación de pobreza si sufre un accidente, pues no está protegido con leyes laborales de previsión social.

Con todo lo anterior se puede plasmar una idea del mundo de la pobreza, conocer desde lo racional de qué hablan los autores cuando se refieren a las personas que viven en situación de pobreza. Sin embargo, estas pierden valor si se oponen a eliminar tal división en la sociedad, si se continúa la discriminación de «los pobres», creyéndolos personas diferentes. Por eso hay que tener en cuenta a quienes viven en pobreza, creer en sus capacidades, interesarse por sus sueños, conocer sus experiencias mediante la real comprensión de lo que significa ser pobre $^{2}$, para lo cual se tienen en cuenta los siguientes aspectos: 1) por tipo de composición de los activos que disponen los hogares; 2) por las estrategias de uso de activos y 3 ) por los que reciben o no asistencia del Estado.

Y, por último, se consideran algunos aspectos clave que se desprenden del enfoque de la vulnerabilidad. En primer lugar, el papel que tienen las políticas públicas en el fortalecimiento o diversificación de los activos físicos, financieros, humanos y sociales de los excluidos pobres, que es el de mayores

Se tienen en cuenta los principales enfoques, métodos y estrategias para la superación de la pobreza Mideplan (2002). 
desventajas sociales. En segundo lugar, el escenario que contribuye a generar el sector público para el acceso al conjunto de oportunidades que brinda la sociedad, en particular mediante las políticas de regulación de los mercados, distribución del ingreso y en los incentivos económicos. En este sentido, el origen de la vulnerabilidad es el rostro opuesto de los activos, es decir, de los pasivos que poseen diversos grupos de población. Los pasivos contribuyen a aumentar los riesgos, exponiendo en mayor medida a los hogares que los poseen. De este modo pueden identificarse políticas públicas que afectan los activos y pasivos, las que pueden contribuir a reducir o no la pobreza, y favorecer o no la integración.

MEDIDAS DE LA POBREZA (NBI, LÍNEA DE POBREZA Y LÍNEA DE INDIGENCIA)

Para evaluar la pobreza se utilizan métodos que miden la calidad de vida y el nivel de ingresos suficientes para subsistir, pero, incluso con esos métodos, no se puede apartar la subjetividad, que significa que toda medición de pobreza debe circunscribirse al lugar y al tiempo; de ahí que según el estándar internacional establecido para el nivel de pobreza, es vivir con menos de dos dólares al día. Ciertamente en los países desarrollados un individuo con un ingreso por encima del promedio mundial también se considera pobre.
Se han propuesto dos tipos de métodos para identificar los pobres y cuantificarlos: el directo y el basado en los ingre$\operatorname{sos}^{3}$. El método directo parte de determinar las múltiples necesidades humanas básicas y fijar normas de satisfacción mínima para cada una de ellas, de acuerdo con el consumo efectivo de las personas u hogares. Quienes se encuentren en situación de subconsumo, según el tipo y grado de insatisfacción de las necesidades que los afecta, son clasificados como pobres.

Para fijar las líneas de pobreza existen, igualmente, diversos enfoques. El primero es el absoluto, que fija el límite normativo a partir de un patrón mínimo de vida, que es valorado de forma tal que permita la satisfacción de las necesidades básicas: nutrición, vivienda, salud, vestuario, etc. En general, la determinación de este límite para reducir el grado de arbitrariedad, empieza por precisar el presupuesto normativo de alimentación que alcance a cubrir los requerimientos nutricionales con una canasta de bajo costo (línea de indigencia).

«En contraste con este enfoque absoluto, el relativo parte del supuesto que existe una relación entre la pobreza y la distribución de ingresos. Destaca la ubicación de los pobres dentro de un contexto de producción y de distribución

\footnotetext{
El método basado en los ingresos consiste en calcular el nivel de estos que se requiere para alcanzar un patrón mínimo de vida y, sobre esta base, se consideran como pobres a quienes perciban un ingreso inferior a ese umbral o línea de pobreza
} 
determinado. Su aplicación conduce, sin embargo, a que la proporción de pobres permanezca constante a lo largo del tiempo» (CEPAL, 1997, p. 13). Para evitar este inconveniente, se ha ensayado la «delimitación de la línea de pobreza en relación a alguna medida de distribución de los ingresos como el promedio de los mismos» (Ministerio de Agricultura, 1989, p. 15).

La cuantificación denota el concepto de pobreza. Esta identifica un grupo de personas que pertenecen a la categoría de pobres. El indicador de necesidades básicas insatisfechas (NBI), uno de los mayormente utilizados, fue introducido por la CEPAL a principios de los años 80. Es la medida más básica, señala la carencia o insuficiencia por parte de un hogar de una de las siguientes cinco necesidades básicas: vivienda con materiales adecuados, acceso a servicios públicos de acueducto y alcantarillado, nivel bajo de hacinamiento (menos de tres personas por cuarto), bajo grado de dependencia (menos de tres personas a cargo de cada trabajador del hogar, con educación) y niños entre los 7 y $\operatorname{los} 11$ años con asistencia escolar idónea. Según este indicador, si el hogar tiene una necesidad insatisfecha se considera que está en condiciones de pobreza, y si tiene más de una, se considera en miseria. Este indicador (NBI) pertenece a la corriente neoclásica con una visión materialista, utilitarista, procedimentalista, cuyas características básicas se relacionan con la falta de un recurso o recursos ante un referente de recursos que posee un individuo, grupo o comunidad.

El Departamento Nacional de Planeación (2002) supone que la medición de la incidencia de la pobreza a través del NBI tiene algunas limitaciones. Considera como pobres a personas que tienen una necesidad básica insatisfecha, pero altos niveles de satisfacción en las necesidades restantes. Además, tres de las cinco variables consideradas dependen de características físicas que pueden estar afectadas por el grado de urbanización más que por las particularidades de los niveles de vida, aunque se definan en forma diferente para la zona urbana y para la rural. Así mismo, el NBI solo permite calcular el porcentaje de personas con una o más necesidades insatisfechas, y no da la posibilidad de tener en cuenta qué tan pobres son los pobres, ni cuál es el grado de desigualdad entre ellos, aspectos que son importantes en materia de política social.

En Colombia se considera población con necesidades básicas insatisfechas (NBI), las viviendas que responden a estos cinco indicadores simples:

1. Vivienda inadecuada: este indicador expresa las características físicas de las viviendas consideradas inapropiadas para el alojamiento humano. Se clasifican en esta situación separadamente las viviendas de las cabeceras municipales y las del resto, así:

- Cabeceras municipales: se incluyen las viviendas móviles, refugio natural 
o puente, aquellas sin paredes o con paredes exteriores de tela, desechos o con piso de tierra.

- Resto: para esta zona se clasifican como inadecuados los mismos tipos anteriores de vivienda. Con relación a los materiales de piso y paredes, solo se consideran en esta situación aquellas que tuvieran un material semipermanente o perecedero (bahareque, guadua, caña o madera), que simultáneamente tengan pisos de tierra.

2. Viviendas con hacinamiento crítico: con este indicador se busca captar los niveles críticos de ocupación de los recursos de la vivienda por el grupo que la habita. Se consideran en esta situación las viviendas con más de tres personas por cuarto.

3. Viviendas con servicios inadecuados: este indicador expresa en forma más directa la falta de acceso a condiciones vitales y sanitarias mínimas. Se distingue, igualmente, la condición de las cabeceras y las del resto. En cabeceras comprende las viviendas sin sanitario o que careciendo de acueducto se provean de agua en río, nacimiento, carrotanque o de la lluvia. En el resto, dadas las condiciones del medio rural, se incluyen las viviendas que carezcan de sanitario y acueducto y que se aprovisionen de agua en río, nacimiento o de la lluvia.

4. Viviendas con alta dependencia económica: es un indicador indirecto sobre los niveles de ingreso. Se clasifican aquí, las viviendas en las cuales haya más de tres personas por miembro ocupado $\mathrm{y}$ que el jefe tenga, como máximo, dos años de educación primaria aprobados.

5. Viviendas con niños en edad escolar que no asisten a la escuela. Mide la satisfacción de necesidades educativas mínimas para la población infantil. Considera las viviendas con, por lo menos, un niño mayor de 6 años y menor de 12 , pariente del jefe y que no asista a un centro de educación formal.

Adicionalmente se tienen en cuenta otros factores para este indicador, como son:

- Urbanización

- Cambio demográfico

- Evolución del capital humano

Dado que cada uno de los indicadores se refiere a necesidades básicas de diferente tipo, a partir de ellos se constituye uno compuesto, que clasifica como pobre o con NBI aquellos hogares que estén, al menos, en una de las situaciones de carencia expresada por los indicadores simples, y en situación de miseria, los hogares que tengan dos o más de los indicadores simples de necesidades básicas insatisfechas. Para estimar la magnitud de la pobreza en relación con la población, se consideró que las personas que habitaban en viviendas con NBI o en miseria se encontraban en las mismas condiciones de su respectiva vivienda. 


\section{LÍNEA DE POBREZA}

Para la estimación de la pobreza, dependiendo del concepto y enfoque que se dé, se han planteado diferentes metodologías. Una de las más utilizadas por organismos internacionales y oficinas de estadística es el método de línea de pobreza, con base en el cual se han suministrado los resultados de pobreza de los estudios estadísticos, obtenidos a partir de la Encuesta de Hogares. El método se aplica solamente a los hogares con ingreso conocido e implica calcular una línea de pobreza que representa el monto mínimo de ingreso que permite a un hogar disponer de recursos suficientes para atender las necesidades básicas. Para ello se requiere contar con:

- El costo de una canasta básica de alimentos (CBA), la cual está formada por un conjunto de alimentos, cuyo contenido calórico y proteico permite satisfacer un nivel mínimo de requerimientos nutricionales por persona al mes. Su costo en determinado periodo de tiempo equivale al ingreso mínimo necesario para satisfacer las necesidades alimentarias de una persona en ese mismo período.

- Con una estimación del costo de las necesidades básicas no alimentarias, según la CEPAL (2001, p. 211), «esta estimación define la canasta normativa explicitando cada satisfactor de forma similar a la CBA»

- Con el ingreso per cápita del hogar.
Se clasifican dentro de la línea de pobreza aquellas personas $u$ hogares que no cuentan con el ingreso mínimo establecido para satisfacer las necesidades básicas. De hecho, para los analistas del tema, la fijación de la línea de pobreza resulta ser una decisión arbitraria; por eso, diferentes autores y organismos utilizan distintas líneas de pobreza. Organismos como el Banco Mundial, proponen líneas de pobreza internacionales en términos de dólares por día por persona ajustadas por PPP. A su vez, los institutos de estadística de cada país suelen publicar líneas de pobreza nacionales (extrema y moderada). Ambos enfoques tienen sus ventajas: mientras las líneas nacionales consideran que existen diferencias entre los países que llevan a identificar a los pobres de distinta manera, las líneas de pobreza internacionales son utilizadas ampliamente porque permiten comparaciones entre países y en el tiempo, así como estimaciones regionales y mundiales de la pobreza.

La línea de pobreza se establece a partir del costo de la canasta básica de alimentos al que se aplica un factor multiplicador.

Así,

$$
\mathrm{LP}=\mathrm{k} \cdot \mathrm{CBA}
$$

Donde: LP es la línea de pobreza, CBA es la canasta básica de alimentos que se define como el conjunto de alimentos expresados en cantidades básicas para satisfacer, por lo menos, las necesidades de calorías (energía) de un individuo 
promedio, de una población de referencia. Es el costo de la canasta básica de alimentos por persona, o línea de indigencia, y k, el factor multiplicador. Así, el valor de la línea de pobreza corresponde a 2 veces el valor de una canasta básica de alimentos, en la zona urbana, y a 1,75 veces, en la zona rural.

La línea de pobreza es estudiada por la corriente neoclásica con una visión utilitarista cuyas características básicas son el nivel mínimo de consumo razonable frente a los estándares de la sociedad. Este método es el más utilizado en la medición de pobreza y se construye con base en la medición de los ingresos y gastos de los hogares, que conduce a una investigación acerca de si los hogares cuentan con los suficientes ingresos para la adquisición de los bienes y servicios indispensables para satisfacer las necesidades básicas. Y para definir estas necesidades se detalla una canasta básica de bienes y servicios que cumpla con los requisitos de satisfacción de necesidades básicas. A partir de esto se obtiene su costo valorando los ítems a precios de mercado. Este costo define la línea: si el ingreso se encuentra por debajo de la línea, el hogar es pobre.

Esta forma de medir la pobreza se ha difundido internacionalmente a través de lo que se conoce como método de línea de pobreza (LP). Con este método se asume que aquellos hogares o individuos cuyos ingresos están por arriba de la línea de pobreza tienen cubiertas sus necesidades básicas.
Es importante resaltar que este método es apropiado para medir tanto la pobreza como la indigencia o pobreza extrema, siendo la distinción entre una y otra medida una variación normativa de la canasta usada para tal fin. En resumen, generalmente, la canasta de indigencia se define a partir de una selección de alimentos que contendrían los nutrientes necesarios para asegurar la subsistencia y evitar la muerte del individuo por inanición. Mientras que la canasta de pobreza incluye los consumos alimentarios agregando una serie de consumos complementarios que teóricamente permitirían cubrir otras necesidades no alimentarias, como las de salud, vestido, vivienda, educación y transporte.

\section{Línea de indigencia}

Según el Banco Mundial, la línea de pobreza -LP- y la línea de indigencia -LI- son propuestas como instrumento de medición. Con este método se compara el ingreso o consumo per cápita con la línea de pobreza expresada en términos per cápita, es por esto que los hogares con ingresos inferiores a la línea de pobreza se consideran pobres.

El indicador permite calcular la incidencia de la pobreza, es decir, el porcentaje de población cuyos ingresos son inferiores a ella. Se calcula como el ingreso mínimo establecido por persona para satisfacer las necesidades alimentarias, de nutrición, vivienda, salud, vestuario y otras necesidades básicas. Corresponde al costo mensual de una canasta básica 
de alimentos por persona, cuyo contenido calórico y proteico permite satisfacer un nivel mínimo de requerimientos nutricionales básicos diarios (2200 calorías, $62 \mathrm{~g}$ de proteínas, calcio, hierro, tiamina, riboflavina, niacina y vitamina C) y que además refleja los hábitos de consumo prevalecientes. Se consideran pobres aquellos cuyo ingreso es inferior a esa línea de pobreza, e indigentes o en extrema pobreza a quienes su ingreso ni siquiera les alcanza para adquirir la canasta básica de alimentos. En este sentido el método provee una aproximación indirecta a la magnitud de la pobreza (Álvarez \& Martínez, 2001).

Para la CEPAL, la «pobreza extrema» o «indigencia» se entiende como la situación en que no se dispone de los recursos que permitan satisfacer al menos las necesidades básicas de alimentación. En otras palabras, se consideran como «pobres extremos» las personas que residen en hogares cuyos ingresos no alcanzan para adquirir una canasta básica de alimentos, así lo destinaran en su totalidad a dicho fin. A su vez, se entiende como «pobreza total», la situación en que los ingresos son inferiores al valor de una canasta básica de bienes y servicios, tanto alimentarios como no alimentarios.

Si bien este indicador comparte su esencia con el empleado oficialmente para monitorear la primera meta del milenio, no coincide necesariamente en su forma de calcularlo. La diferencia fundamental radica en que el umbral de suficiencia en el indicador oficial está definido en términos de un valor en dólares, idéntico para todos los países en términos de paridad del poder de compra. En cambio, las canastas básicas que dan lugar a las líneas utilizadas por la CEPAL son específicas para cada país y respetan las estructuras de consumo prevalecientes en ellos (Organización de las Naciones Unidas, 2010).

Con referencia a los indicadores como tales, al indicador NBI se le atribuye el problema que las cinco necesidades que mide inciden mayormente entre los clasificados como pobres que entre la población en conjunto, por lo que la incidencia entre las personas de bajos ingresos no es angustiosa. Según el Banco Mundial (2000), el $80 \%$ de las personas clasificadas como pobres tiene ingresos superiores a aquellas que son pobres de acuerdo con la línea de pobreza (LP), y esto conlleva implicaciones distributivas porque de esta manera se les estará ayudando menos a aquellos individuos con menores ingresos.

\section{POLITÍCAS PÚBLICAS PARA LA SUPERACIÓN DE LA POBREZA}

Este tema de las políticas públicas se define como la esencia de las acciones que son ejecutadas por los gobernantes cuyo objetivo es generar bienestar a toda la sociedad. Estas políticas están encaminadas a dar solución a una serie de necesidades sociales, donde se busca la concentración de esfuerzos en sectores de vital importancia para el Estado, como lo son la educación, la salud, la 
vivienda y el empleo, ya que permiten otorgar al individuo un cierto grado de certidumbre buscando con esto llevarlo a un bienestar colectivo. Por eso la política social incluye otros aspectos como son distribución, protección y justicia social. Sin embargo, y contrario a todo lo anterior, las políticas públicas son frecuentemente capturadas por las élites y los grupos que tienen el poder, que se benefician desproporcionadamente de ellas, sumergiendo a una gran población por debajo de la línea de la pobreza de dos dólares al día. La ausencia de compromiso en la gestión de políticas públicas, la carencia de oportunidades para una mayoría, las grandes desigualdades y la pobreza, conllevan la desintegración social y la violencia. No es desconocido que la capacidad de un país para generar mayores ingresos contribuye con la mejora de los estándares de vida de una población, pero, por sí sola no garantiza que haya una equitativa distribución y resultados favorables que proporcionen progreso a una región y que favorezcan a toda su población. Para que se logre lo anterior se le da un papel importante a las políticas sociales, pero para ello deben ser eficientes y sustentables. Estas políticas deben abarcar tanto el apoyo para la satisfacción de las necesidades básicas de la población más carente, como la creación de mayores oportunidades de empleo para la capacidad de generación de ingresos propios.

Una política del Gobierno que sea integral, debe tener por lo menos dos objetivos, reducción de desigualdades y crecimiento económico, focalizándose en las regiones que evidencian persistencia de la pobreza. Los gobiernos utilizan la política social como herramienta para organizar $y$ perfeccionar las instituciones del mercado y el sistema social. Mediante el buen uso de las políticas sociales se puede lograr acabar con el círculo vicioso de la pobreza, y crear un círculo bondadoso en el que el desarrollo humano y el empleo generen una mayor demanda interna $\mathrm{y}$, por ende, crecimiento económico.

Para desarrollar las políticas públicas se necesitan estrategias basadas en transparencia, honestidad y eficiencia, donde el interés general esté por encima del particular. Es este reconocimiento el que hace que la labor sea eficaz y permita cumplir su finalidad llegando a la población vulnerable sin caer en el egoísmo de satisfacer el interés de unos pocos. Para evitar esto debemos exigir que el Estado cumpla con las políticas públicas; que estas se efectúen como deben ser y además exigir el manejo óptimo de los recursos. Solo de esta manera seremos un Estado social de derecho, donde prime la igualdad y la libertad para todos.

Autores como Meny y Thoenig (1989), desde una perspectiva analítica, conceptúan las políticas públicas como «los programas de acción de una autoridad pública en un sector de la sociedad o en un espacio geográfico» (Menyy \& Thoenig, 1989, p. 19).

Adicionalmente, Anderson ofrece una visión genérica: «Una orientación delibe- 
radamente seguida por un actor o por un grupo de actores al tratar un problema o una cuestión que les concierne» (Anderson, 1990, p. 30).

Las anteriores definiciones indican que el actor que produce una política pública es (o está habilitado para actuar como), un actor público y, por ende, a pesar de que otro tipo de actor social puede influenciar las acciones de los gobiernos, no puede producir estas políticas. Además señalan que una política pública puede ser producto de una decisión al igual que de la omisión de tomar una decisión. Finalmente indican que las políticas públicas pueden ir desde la propuesta de erradicar la pobreza, hasta la de evitar que se incremente, pasando por una graduación de objetivos y medios para alcanzarlos.

En el caso colombiano, los gobiernos de turno establecen programas de política social en forma coyuntural, para dar solución al problema de la pobreza; pero dichas políticas ignoran los problemas principales -en una visión macroeconómica y política- como lo son la inequidad en la distribución de los ingresos, el acceso a recursos que conducen una sociedad, lo cual lleva a mantener las inequidades, principalmente en lo referente a la posibilidad de los individuos y las familias de tener acceso a mejores asignaciones de recursos (bienes públicos), en este caso: empleo, capital humano, vivienda, salud, entre otros, que ayuden a competir en los mercados de trabajo.
Los programas sociales por parte del Gobierno se basan en valores sesgados que desvirtúan el origen de la pobreza, y la política social, como se ha concebido, se torna en un atenuante de las consecuencias que a corto plazo provoca la aplicación de las medidas macroeconómica de ajuste.

\section{CONCLUSIONES}

Muchos son los conceptos sobre pobreza que no hacen referencia solamente a la falta de ingresos económicos, a la privación o falta de acceso a ciertos bienes y servicios, considerados necesarios por la sociedad, sino que incluyen diferentes aspectos, como vivienda adecuada, salud, acceso a servicios públicos, educación, vestido, esparcimiento, etc., necesarios también para obtener bienestar.

La relación entre las teorías del desarrollo, los enfoques de los derechos humanos y los problemas concretos a los que alude el concepto de pobreza, van configurando un marco de interpretación crecientemente aceptado por la comunidad internacional. No obstante, la mayor parte de los esfuerzos destinados a obtener mediciones de la pobreza continúan restringiéndose a la consideración de un grupo de indicadores económicos, como el de ingresos insuficientes.

De acuerdo con la medición a través de las necesidades básicas insatisfechas, 
la solución para la disminución de la pobreza sería incrementar el gasto social y aumentar el cubrimiento de los servicios públicos y la educación, pero esto podría ser una falacia, pues una vez se terminen los programas de asistencia pasará muy poco tiempo para que los individuos vuelvan al estado de pobreza, medido por la línea de pobreza, por lo cual, la mejor forma de erradicar la pobreza es a través de una política que no solo incremente el cubrimiento de los servicios sociales sino que también incentive a las personas a mejorarse a sí mismas.
Se le debe dar importancia real al problema de la pobreza, estudiarla y conocerla más para poder proponer alternativas de solución, con el objetivo de erradicarla o por lo menos disminuirla, involucrando a la sociedad e instituciones sociales.

Las políticas públicas que aplican los gobiernos, que incluyen distintos programas para dar solución a los problemas de la pobreza llevando a la población a un «bienestar», están encaminadas a la disminución de una serie de necesidades que azotan a una parte de la población.

\section{REFERENCIAS}

Álvarez, M. E. \& Martínez, H. (2001). El desafio de la pobreza. Bogotá: Fundación Social.

Anderson, J.E. (1990). Public policymaking: an introduction. Boston, MA: HoughtonMifflin Co.

Banco Mundial. (1990). World Develoment Report: poverty. Washington DC: WB.

Banco Mundial. (2002). Informe sobre la pobreza 2000-2001. Recuperado de ww.worldbank.org/poverty.

Becker, G. (1983). El capital humano. (2 ed.). Madrid: Alianza.

Boltvinik, J. (2010). Principios de medición multidimensional de la pobreza. México: Instituto de Investigaciones Económicas.

Comisión Económica para América Latina y el Caribe-CEPAL- (1997). La brecha de la equidad: América Latina, el Caribe y la Cumbre Social. Santiago de Chile: CEPAL. 
Comisión Económica para América Latina y el Caribe-CEPAL- (2001). Seminario Internacional. Las diferentes expresiones de la vulnerabilidad social en América Latina y el Caribe. Santiago de Chile.

Departamento Nacional de Planeación. (2006). Los compromisos fundamentales de la sociedad: reconstitución del tejido social. Bogotá: DNP.

Dirección de Desarrollo Social -DDS- (2011). Seminario: Diálogos de protección social. La protección social argentina en perspectiva latinoamericana: retos de inclusión e integralidad, Argentina.

Informe sobre el Desarrollo Mundial. (2000). Lucha contra la pobreza. Panorama General.

Menyy, Y. \& Thoenig, J.C. (1989). Les Politiques Publiques. Paris: PUF.

Ministerio de Planificación y Cooperación -MIDEPLAN-. (2002). Perspectivas innovativas en politica social. Experiencias relevantes para la superación de la pobreza: una mirada desde lo posible. Chile.

Nussbaum, M. (2002) Las mujeres y el desarrollo. Buenos Aires.

Organización de las Naciones Unidas ONU. (1995). Cumbre Mundial sobre Desarrollo Social. Copenhague, Dinamarca.

Organización de las Naciones Unidas ONU. (2010). El progreso de América Latina y el Caribe hacia los objetivos del milenio. NY: ONU.

Programa de las Naciones Unidas para el Desarrollo -PNUD- (1997). La pobreza en la perspectiva del desarrollo humano: concepto y medición. Nueva York: PNUD.

Programa de las Naciones Unidas para el Desarrollo -PNUD- (1997). Pobreza y desarrollo humano. New York: PNUD.

Pogge, T. (2005). Severe poverty as a violation of negative duties. Ethics and International Affairs, 19(1), 55-84.

Sen, A. (1996). Capacidad y bienestar. México: FCE.

Sen, A. (2009). Sobre conceptos y medidas de pobreza. Comercio Exterior, 42. 